\title{
Efetividade do programa de controle ao tabagismo em uma cidade do sul do Brasil
}

\author{
Effectiveness of the tobacco control program in a city of the south of Brazil \\ Efectividad del programa de control al tababismo en una ciudad del sur del Brasil \\ Kelser de Souza Kock ${ }^{a}$, Matheus Walerio Braido Arantes ${ }^{a}$, Carolina Campos dos Santos ${ }^{b}$, Solange de Souza Castelini ${ }^{b}$
}

\section{Resumo}

Objetivo: Avaliar a efetividade do programa de controle ao tabagismo em uma cidade do sul do Brasil. Métodos: Foi realizado um estudo de Coorte Retrospectiva que analisou os resultados das unidades de saúde que participaram do Programa de Controle do Tabagismo na cidade de Tubarão (SC) no período de 2014 a 2016. Resultados: Foram analisados dados de 106 usuários, sendo na maioria mulheres, com média de idade de 51,0 $\pm 10,9$ anos. A efetividade do programa foi de $51,9 \%$, com tempo médio para cessação tabágica de aproximadamente de 3 semanas. Os indivíduos do sexo feminino e que participaram de menos do que 4 encontros apresentaram maior dificuldade em parar de fumar. Conclusão: $O$ programa apresenta boa efetividade, compatível com outros programas semelhantes no Brasil e no exterior. A dependência à nicotina e uso de bupropiona não foram fatores determinantes no desfecho e o período em que o programa foi mais efetivo quanto à cessação do tabaco foram as 3 semanas iniciais.

\section{Abstract}

Objective: To evaluate the effectiveness of the tobacco control program in a city in southern Brazil. Methods: A retrospective cohort study was carried out, which analyzed the results of the Health Units that participated in the Smoking Control Program in the city of Tubarão (SC), from 2014 to 2016. Results: Data from 106 participants were analyzed, mostly women with mean age of $51.0 \pm 10.9$ years. The effectiveness of the program was $51.9 \%$, with a mean time for smoking cessation of approximately three weeks. Female Individuals who participate in less than four meetings present greater difficulty in quitting smoking. Conclusion: The program has good efficacy, compatible with other similar programs in Brazil and exterior. Nicotine dependence and use of bupropion were not determinant factors in the outcome and the period when the program was most effective in tobacco cessation were the first three weeks.

\section{Resumen}

Objetivo: Evaluar la efectividad del programa de control al tabaquismo en una ciudad del sur de Brasil. Métodos: Se realizó un estudio de Cohorte Retrospectiva que analizó los resultados de las unidades de salud que participaron del Programa de Control del Tabaquismo en la ciudad de Tubarão (SC) en el período de 2014 a 2016. Resultados: Se analizaron datos de 106 participantes, siendo en su mayoría Mujeres con una media de edad de 51,0 $0 \pm 10,9$ años. La efectividad del programa fue del $51,9 \%$, con tiempo promedio para cesación tabáquica de aproximadamente de 3 semanas. Las personas del sexo femenino y que participaron en menos de 4 encuentros presentaron mayor dificultad para dejar de fumar. Conclusión: El programa presenta buena efectividad, compatible con otros programas similares en Brasil y en el exterior. La dependencia a la nicotina y el uso de bupropión no fueron factores determinantes en el desenlace y el período en que el programa fue más efectivo en cuanto al cese del tabaco fueron las 3 semanas iniciales.

Como citar: Kock KS, Arantes MWB, Santos CC, Castelini SS. Efetividade do programa de controle ao tabagismo em uma cidade do sul do Brasil. Rev Bras Med Fam Comunidade. 2017;12(39):1-10. http://dx.doi.org/10.5712/rbmfc12(39)1562
Palavras-chave:

Hábito de Fumar

Abandono do Uso de Tabaco

Programa Nacional de

Controle do Tabagismo

Atenção Primária à Saúde
Keywords:

Smoking

Tobacco Use Cessation

National Program of

Tobacco Control

Primary Health Care
Palabras clave:

Hábito de Fumar

Cese del Uso de Tabaco

Programa Nacional de

Control del Tabaquismo

Atención Primaria de Salud
Fonte de financiamento:

declaram não haver.

Parecer CEP:

1.938.161 (UNISUL), aprovado

em 22/02/2017.

Conflito de interesses:

declaram não haver.

Procedência e revisão por pares: revisado por pares.

Recebido em: 07/07/2017.

Aprovado em: 22/01/2018. 


\section{Introdução}

O tabagismo é a principal causa global prevenível de morbidade e mortalidade, além de ser um dos mais importantes fatores de risco para as doenças crônicas não transmissíveis, responsável por cerca de 6 milhões de mortes todos os anos. As projeções apontam que, em 2020, esse número será de 7,5 milhões, ou seja, $10 \%$ de todas as mortes ocorridas no mundo. ${ }^{1}$ No Brasil existem cerca de 22 milhões de fumantes que necessitam de tratamento para cessar o tabagismo, além de aproximadamente 26 milhões de ex-fumantes que pararam de fumar por conta própria ou com tratamento. ${ }^{2}$

De acordo com dados da Organização Mundial de Saúde (OMS), há evidências epidemiológicas que apontam uma relação de causalidade entre o tabagismo e cerca de 50 doenças crônicas não transmissíveis: doenças cardiovasculares, acidente vascular cerebral (AVC), doença pulmonar obstrutiva crônica (DPOC), câncer (no pulmão e em outros sítios). Estima-se que o fato de nunca ter fumado ou deixar de fumar reduza o risco em desenvolver essas doenças em até 30\%, chegando à redução de até 90\% para o câncer de pulmão e DPOC. ${ }^{3,4}$

Apesar de um declínio anual de fumantes em torno de 3-4\%, devido principalmente às políticas pós-Convenção-Quadro sobre o Controle do Tabaco (FCTC) da OMS como política de preços, proibição do fumo interno, dentre outras, o lucro anual obtido com a venda de cigarros no mundo é de US\$ 85 bilhões para a indústria do tabaco. ${ }^{2} \mathrm{Em}$ contrapartida, algumas estimativas conservadoras indicam que os custos em saúde com doenças relacionadas ao tabaco alcancem US $\$ 500$ bilhões por ano. ${ }^{5}$

Há alguns anos, políticas mundiais foram desenvolvidas com o objetivo de combater o tabagismo tentando prevenir seu início e promovendo a cessação de fumar por meio da Convenção Quadro da Organização Mundial da Saúde para Controle do Tabaco (CQCT/OMS) da qual o Brasil é Estado-Parte desde 2005. Atuando como referência e liderança, o Programa Nacional de Controle do Tabagismo, já existia no país desde 1989, o qual passou a ser responsável pela internalização no Sistema Único de Saúde orientando e cumprindo as diretrizes da CQTC pelo país. ${ }^{6}$

A estratégia da CQTC se apoia em seis pilares principais como medidas efetivas para o controle do tabaco conhecidos pela sigla MPOWER: [M] - Monitoramento, [P] - Proteção de não fumantes, [O] - Oferta de tratamento para deixar de fumar, [W] - Advertência quanto aos perigos do tabaco, [E] - Proibição de propaganda, promoção e patrocínio, e [R] - Aumento de impostos. ${ }^{7}$

No Brasil, o Instituto Nacional de Câncer José Alencar Gomes da Silva (INCA) articula uma rede com representantes de Secretarias de Educação e Saúde, Estaduais e Municipais, de todos os estados e cerca de 3000 municípios que são responsáveis pelo desenvolvimento de ações de controle do tabaco em suas esferas. Nos anos de 2011 e 2012 o controle do tabaco foi incluído no Plano Estadual de Saúde de 24 Estados e é considerado prioridade de governo em 17 deles, fortalecendo o controle do tabagismo em seus municípios. ${ }^{7}$

Nos municípios, o tratamento do tabagismo deve ser realizado por meio da abordagem cognitivo-comportamental obrigatória e apoio medicamentoso quando indicado, e pode ser realizado por qualquer unidade de saúde pertencente ao SUS, de qualquer nível hierárquico, segundo critério do gestor municipal, desde que preencha os critérios de credenciamento definidos pelo Plano de Implantação. ${ }^{8}$ 
O programa de controle do tabagismo atualmente utilizado no Brasil propõe diretrizes para a terapêutica do fumante. Inicialmente, é realizada uma avaliação com questionário padrão sobre comorbidades, história tabágica, grau de dependência de nicotina com uso do teste de Fagerström e então se propõe um plano terapêutico. Após essa avaliação inicial, o usuário é inserido em grupos de terapia cognitivo-comportamental, sendo que as quatro primeiras sessões são semanais. O passo seguinte é o apoio medicamentoso aos usuários com alto nível de dependência, realizado por meio do acesso gratuito a gomas de mascar, adesivos de nicotina e bupropiona, além de material educativo para os coordenadores das sessões e para os usuários fumantes. ${ }^{9}$

Apesar de sofrer variações pelas características ambientais e humanas na qual é aplicado, estudos mostram que a taxa de sucesso na cessação do tabagismo é de cerca de $80 \%$ após as quatro sessões iniciais de terapia cognitivo-comportamental e entre $60-66 \%$ permanecem abstinentes após o término do tratamento. ${ }^{10-12}$

Sabe-se que existem poucos estudos no Brasil sobre os resultados e a eficiência das estratégias propostas nos programas de controle do tabagismo e esse número é ainda menor quando se trata das características particulares dos municípios. Sabe-se que as variações provocadas pelas características ambientais e humanas da microrregião onde o programa é aplicado interferem na taxa de sucesso da cessação do tabagismo, o que torna indispensável que estudos relacionados ao tema sejam conduzidos em nível de município. ${ }^{6-8}$

Neste contexto, o presente estudo visa avaliar a efetividade do programa de controle do tabagismo no município de Tubarão (SC) de modo a obter o reconhecimento das variáveis mais importantes para a taxa de sucesso do tratamento. Espera-se por meio da análise detalhada das variáveis propor modificações em determinadas abordagens do programa, bem como intensificar métodos que apresentem resultado satisfatório.

\section{Métodos}

\section{Tipo de estudo}

Foi realizado um estudo de Coorte Retrospectiva.

\section{Local do estudo}

Na cidade de Tubarão (SC), o sistema de sáude é composto por 29 equipes de Estratégias de Saúde da Família (ESF), um Programa de Agente Comunitário de Saúde (PACS), uma Policlínica Central, uma Clínica de Referência, um Centro de Atendimento Psicossocial (CAPS) II, um CAPS AD, um Centro de Atendimento Especializado (CAES) e três Núcleos de Apoio à Saúde da Família (NASF).

As seguintes unidades de saúde que participaram do Programa de Controle do Tabagismo na cidade de Tubarão-SC: Policlínica Central, ESF Humaitá de Cima, ESF KM 60, CAPS AD e ESF Campestre.

População do estudo

Foram estudados os dados referentes aos usuários fumantes de cinco unidades de saúde que participaram do Programa de Controle do Tabagismo na cidade de Tubarão-SC no período de 2014 a 2016. 
Características regionais do programa de controle ao tabagismo em Tubarão (SC)

O programa é realizado na atenção básica, de acordo com a adesão e disponibilidade da equipe de ESF e CAPS AD, no período diurno e, de forma centralizada, na Policlínica, no período noturno. A duração do programa é de 120 dias, organizados da seguinte forma:

- Contato inicial: Abordagem dos interessados na cessação tabágica;

- Quatro encontros estruturados semanais;

- Dois encontros estruturados quinzenais;

- Dois encontros estruturados mensais de manutenção.

Nos encontros são realizadas abordagens comportamentais e medicamentosas conforme as ações indicadas pelo INCA e Ministério da Saúde.

As medicações disponibilizadas são goma de mascar e adesivos com nicotina e a bupropiona, indicadas ao usuário conforme avaliação da equipe.

\section{Método de coleta de dados}

Foi realizado contato com o coordenador municipal do programa de controle ao tabagismo, para solicitação da planilha de acompanhamento das sessões. Nesta planilha, constam nome do participante usuário (excluído quando armazenado e exportado para planilha de análise estatística), idade, sexo, medicações utilizadas, frequência e horário dos encontros, nível de dependência à nicotina, avaliada pelo questionário de Fagerström, e data da cessação tabágica nos casos de sucesso.

O questionário de Fagerström apresenta uma pontuação de 0 a 10, em que 0 a 4 é considerada dependência leve, 5 a 7 dependência moderada e 8 a 10 dependência grave ${ }^{6}$ Nos casos de cessação e recaída no período dos 120 dias do programa, foi considerado insucesso. Todo o processo de coleta foi realizado em abril de 2017.

Critérios de inclusão

Foram incluídos somente os usuários das unidades de saúde referidas.

\section{Critérios de exclusão}

Foram excluídos os casos com ausência da informação de sucesso ou insucesso na cessação do tabagismo ao final do programa.

\section{Análise estatística}

Os dados foram armazenados em um banco de dados criado com o auxílio do software Microsoft Excel ${ }^{\circledR}$, e posteriormente exportados para o software SPSS $20.0^{\circledR}$. Os mesmos foram apresentados por meio de números absolutos e percentuais ou medidas de tendência central e dispersão, dependendo do tipo de variável. Foi realizada análise bivariada e multivariada por meio de regressão logística bruta e ajustada dos preditores em relação ao desfecho insucesso, para obtenção do odds ratio. Foi considerado intervalo de confiança de 95\%, com nível de significância estatística de 5\%. 


\section{Aspectos éticos}

Este estudo foi submetido ao Comitê de Ética em Pesquisa da Unisul (CEP - Unisul) e após a sua aprovação iniciou-se a coleta de dados, sendo respeitados os preceitos da resolução 466/2012 do Conselho Nacional de Saúde. O número de parecer de aprovação foi 1.938.161 e o CAAE 64339317.0.0000.5369. Conforme o parecer do CEP, não foi necessária a assinatura do Termo de Consentimento Livre e Esclarecido (TCLE), pois os usuários já haviam finalizado sua participação no programa.

\section{Resultados}

Foram coletados dados de 130 usuários do prorgama de controle ao tabagismo, sendo 24 excluídos deles por ausência da informação sucesso ou insucesso nas planilhas de acompanhamento. Dentre estes, estavam os usuários da ESF Campestre e do CAPS AD, que não possuíam esta informação e foram

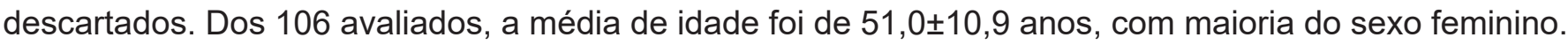
Os medicamentos mais utilizados pelos usuários foram o adesivo de nicotina e a goma de mascar.

A maioria dos usuários frequentaram as reuniões na policlínica durante o período noturno. Mais da metade dos usuários participaram de quatro ou mais reuniões do programa de controle ao tabagismo. A classificação mais prevalente de dependência à nicotina foi moderada, conforme escala de Fagerström e aproximadamente metade dos usuários deixou de fumar neste período (Tabela 1).

Tabela 1. Características da amostra.

\begin{tabular}{|c|c|c|}
\hline & $\mathbf{N}$ & $\%$ \\
\hline \multicolumn{3}{|l|}{ Sexo } \\
\hline Masculino & 37 & 34,9 \\
\hline Feminino & 69 & 65,1 \\
\hline \multicolumn{3}{|l|}{ Medicamentos } \\
\hline Adesivo & 103 & 97,2 \\
\hline Goma & 84 & 79,2 \\
\hline Bupropiona & 39 & 36,8 \\
\hline \multicolumn{3}{|l|}{ Local } \\
\hline Humaitá de cima (diurno)/2014B & 11 & 10,4 \\
\hline Km 60 (diurno/2015B & 17 & 16,0 \\
\hline Policlínica (noturno)/2015B & 32 & 30,2 \\
\hline Policlínica (noturno)/2016A & 19 & 17,9 \\
\hline Policlínica (noturno)/2016B & 27 & 25,5 \\
\hline \multicolumn{3}{|l|}{ Dependência à nicotina } \\
\hline Leve & 22 & 21,0 \\
\hline Moderada & 58 & 55,2 \\
\hline Grave & 25 & 23,8 \\
\hline \multicolumn{3}{|l|}{ Encontros } \\
\hline Até 4 & 57 & 43,8 \\
\hline Mais que 4 & 73 & 56,2 \\
\hline \multicolumn{3}{|l|}{ Desfecho } \\
\hline Sucesso & 55 & 51,9 \\
\hline Insucesso & 51 & 48,1 \\
\hline
\end{tabular}


Analisando os fatores relacionados ao desfecho na cessação do tabagismo, foi observado que usuários do sexo feminino tiveram mais dificuldade de parar de fumar e usuários que frequentaram mais que quatro encontros tiveram melhores resultados na cessação tabágica. Dentre os medicamentos avaliados, foram descartados da análise a goma e o adesivo, pois estiveram presentes em grande parte da amostra. Apenas a bupropiona foi mantida na análise. O tempo médio para cessação foi de 19,2 $\pm 20,9$ dias. Maiores informações podem ser visualizados na Tabela 2, onde são comparados os grupos sucesso e insucesso e analisados o odds ratio bruto e ajustado das variáveis preditoras do insucesso.

Tabela 2. Comparação dos grupos sucesso e insucesso para cessação do tabagismo e análise do odds ratio (OR) bruto e ajustado das variáveis preditoras do insucesso

\begin{tabular}{|c|c|c|c|c|c|c|}
\hline & $\begin{array}{c}\text { Sucesso } \\
N=55 \\
(51,9 \%) \\
n(\%)\end{array}$ & $\begin{array}{c}\text { Insucesso } \\
N=55 \\
(48,1 \%) \\
n(\%)\end{array}$ & $\begin{array}{l}\text { OR BRUTO } \\
\text { (IC 95\%) }\end{array}$ & $\mathbf{p}$ & $\begin{array}{l}\text { OR AJUSTADO } \\
\text { (IC 95\%) }\end{array}$ & $\mathbf{p}$ \\
\hline \multicolumn{7}{|l|}{ Sexo } \\
\hline Homem & $24(64,9)$ & $13(35,1)$ & 1,00 & & 1,00 & \\
\hline Mulher & $31(44,9)$ & $38(55,1)$ & $2,263(1,000-5,164)$ & 0,050 & $4,002(1,293-12,391)$ & 0,016 \\
\hline \multicolumn{7}{|l|}{ Idade (anos) } \\
\hline$\geq 18$ e $<40$ anos & $11(50,0)$ & $11(50,0)$ & 1,00 & & 1,00 & \\
\hline$\geq 40$ e $<60$ anos & $33(54,1)$ & $28(45,9)$ & $0,848(0,320-2,251)$ & 0,741 & $0,552(0,159-1,916)$ & 0,350 \\
\hline$\geq 60$ anos & $11(47,8)$ & $12(52,2)$ & $1,091(0,339-3,513)$ & 0,884 & $1,915(0,448-8,193)$ & 0,381 \\
\hline \multicolumn{7}{|l|}{ Encontros } \\
\hline Até 4 & $14(30,4)$ & $32(69,6)$ & 1,00 & & 1,00 & \\
\hline Mais que 4 & $41(68,3)$ & $19(31,7)$ & $0,203(0,088-0,465)$ & $<0,001$ & $0,101(0,032-0,318)$ & $<0,001$ \\
\hline \multicolumn{7}{|l|}{ Local } \\
\hline Humaitá de cima/2014B & $6(54,5)$ & $5(45,5)$ & 1,00 & & 1,00 & \\
\hline $\mathrm{Km} \mathrm{60/2015B}$ & $8(47,1)$ & $9(52,9)$ & $1,350(0,295-6,183)$ & 0,699 & $1,473(0,255-8,524)$ & 0,665 \\
\hline Policlínica/2015B & $17(53,1)$ & $15(46,9)$ & $1,059(0,268-4,188)$ & 0,935 & $2,317(0,409-13,115)$ & 0,342 \\
\hline Policlínica/2016A & $8(42,1)$ & $11(57,9)$ & $1,650(0,379-7,365)$ & 0,512 & $2,391(0,395-14,454)$ & 0,342 \\
\hline Policlínica/2016B & $16(59,3)$ & $11(40,7)$ & $0,825(0,201-3,391)$ & 0,790 & $0,583(0,085-4,000)$ & 0,583 \\
\hline \multicolumn{7}{|l|}{ Período } \\
\hline Diurno & $14(50,0)$ & $14(50,0)$ & 1,00 & & 1,00 & \\
\hline Noturno & $41(52,6)$ & $37(47,4)$ & $0,902(0,380-2,141)$ & 0,816 & $1,315(0,444-3,895)$ & 0,621 \\
\hline \multicolumn{7}{|l|}{ Dependência à nicotina } \\
\hline Leve & $10(45,5)$ & $12(54,5)$ & 1,00 & & 1,00 & \\
\hline Moderada & $29(50,0)$ & $29(50,0)$ & $0,833(0,311-2,230)$ & 0,717 & $1,853(0,556-6,174)$ & 0,315 \\
\hline Grave & $16(64,0)$ & $9(36,0)$ & $0,469(0,145-1,512)$ & 0,205 & $0,917(0,215-3,915)$ & 0,907 \\
\hline \multicolumn{7}{|l|}{ Bupropiona } \\
\hline Utilizando & $24(61,5)$ & $15(38,5)$ & 1,00 & & 1,00 & \\
\hline Não utilizando & $31(46,3)$ & $36(53,7)$ & $1,858(0,831-4,153)$ & 0,131 & $0,551(0,194-1,563)$ & 0,263 \\
\hline
\end{tabular}




\section{Discussão}

Resumo dos principais achados do estudo

Os principais achados do presente estudo foram em relação à caracterização da população usuário do programa de controle ao tabagismo como sendo em sua maioria mulheres de meia idade, com dependência moderada à nicotina. Aproximadamente metade dos usuários conseguiram parar de fumar e com relação às características do programa, pode-se afirmar que sua maior efetividade ocorreu na fase inicial, com pico de cessação nas três primeiras semanas e que uma maior frequência nos encontros presenciais foi fundamental para o sucesso do programa.

Fortalezas e limitações do estudo

O presente estudo apresenta como fortalezas a análise de mais de dois anos de características regionais do programa de controle ao tabagismo, com informações sobre os resultados e associações de sua efetividade. Como limitação do estudo, pode-se citar a utilização de dados secundários na coleta de dados e a confirmação da cessação do tabagismo por autorrelato.

Ainda, deve-se comentar a perda amostral dos dados de 24 usuários da ESF Campestre e do CAPS $A D$, que não possuíam a informação sobre o desfecho. Apesar de reduzir o poder estatístico, pondera-se que a ausência destes dados tenha pouca intereferência nos resultados do trabalho, pois nos grupos avaliados não houve diferença na cessação tabágica.

Comparação com a literatura já existente

De acordo com os resultados deste estudo, os usuários eram na maioria do sexo feminino de meia idade. $O$ estudo de Rodrigues et al., ${ }^{13}$ realizado no Brasil, corrobora esses achados pois também analisou usuários de meia idade, sendo $71 \%$ constituído por mulheres. Outro estudo, avaliando tabagistas chilenos, de Peña et al.. ${ }^{14}$ apresentou resultados divergentes quanto ao sexo dos usuários, pois obteve $65 \%$ dos usuários do sexo masculino, que foram acompanhados durante um ano com entrevistas via telefone e demonstrou um relação direta entre cessação do tabagismo e nível de escolaridade. Estes dados demonstram que as características do programa de controle ao tabagismo podem divergir quando ao modelo de atuação e adesão dos usuários.

O nível de dependência à nicotina dos avaliados foram similares aos de Rodrigues et al., ${ }^{13}$ mas divergiu na caracterização da alta dependência pelo teste de Fagerström ser um fator determinante no insucesso, o presente estudo não apresentou significância estatística quanto à esse fator.

Os medicamentos utilizados no programa de controle ao tabagismo foram diferentes dos utilizados por Peña et al.., ${ }^{14}$ que utilizaram como principal droga vareniclina no programa chileno, enquanto o programa em Tubarão utiliza bupropiona como principal droga. Vale ressaltar que a escolha de cada droga está relacionada a disponibilidade, custos, e sua escolha varia de acordo com cada local. 
Muitos são os modelos de programas de controle ao tabagismo pelo mundo, podendo-se citar a utilização da internet, como apresentado por Mavrot et al., ${ }^{15}$ a via telefonônica como Peña et al. ${ }^{14}$ ou com reuniões em grupo presenciais, como é o programa da pesquisa em questão. Esses modelos se diferenciam na forma de influenciar e conscientizar o fumante a cessar o uso do tabaco, porém apresentam resultados semelhantes quanto a obtenção do objetivo final.

O presente estudo apresentou uma taxa de sucesso semelhante aos estudos Rodrigues et al. ${ }^{13}$ $(51,7 \%)$ e Peña et al. ${ }^{14}$ (53,4\%), entretanto diverge dos resultados de Mavrot et al. ${ }^{15}$ que, por meio de um estudo randomizado utilizando a internet, apresentou taxa de cessação de tabagismo de, aproximadamente, $20 \%$ após 3 meses do programa. Então, pode-se ponderar que programas com encontros presenciais são mais efetivos na cessação ao tabagismo.

O tempo médio para os usuários conseguirem deixar de fumar esteve compreendido nas primeiras três semanas, neste programa, cuja duração total é de 120 dias. Este achado demonstra que a eficácia da cessação tabágica é maior no início do programa.

Sabe-se que os homens são os que apresentam os melhores resultados quanto à cessação do tabagismo, como demonstrado no presente estudo e corroborado pelos estudos Rodrigues et al. ${ }^{13}$ e Peña et al. ${ }^{14}$ Este resultado pode ser explicado pelo fato de que as mulheres tendem a utilizar o cigarro com uma ferramenta de autoafirmação ancorando a imagem da mulher madura, adulta e segura de si no ato de fumar, além disso, de certa forma a mulher tende a utilizar mais o cigarro como apoio para enfrentar as dificuldades diárias, problemas no trabalho, estresse, problemas familiares, dificultando ainda mais o processo de cessação do tabaco como é visto por Jesus et al. ${ }^{16}$ e Eckerdt e Corradi-Webster. ${ }^{17}$

A adesão ao programa se traduz pelo número de encontros que cada usuário frequentou e, no presente estudo, esse número foi associado à taxa de sucesso em deixar de fumar. Este achado parece demonstrar que a maior adesão ao programa pode ser um fator preditivo na cessação do tabagismo.

Em relação ao efeito da faixa etária e grau de dependência, não foram encontradas associações significativas com o sucesso no programa. Estes resultados divergem do encontrado no estudo feito por Figueiró et al. ${ }^{18}$ e do estudo de Curry et al., ${ }^{19}$ que apresentaram diferenças na população participante dos mesmos, com maior médias de idade e baixa dependência à nicotina, como fatores preditivos de sucesso.

Outros fatores não se mostraram associados à cessação do tabagismo, como a participação em programas noturnos ou diurnos e uso de bupropiona. Esperava-se que a influência desses fatores fossem maiores, a praticidade do horário noturno para os trabalhadores e o uso de uma medicação associada facilitaria o processo de parar de fumar, como apresentados por França et al. ${ }^{20}$ e por Mavrot et al. ${ }^{15}$.

Implicações para a pesquisa na área e/ou para a prática dos profissionais

As implicações são amplas, tanto para novas pesquisas que possam detalhar resultados encontrados e investigar motivos de baixa efetividade na parte final do programa, por exemplo, quanto para que as autoridades responsáveis pelo programa no âmbito municipal tomem condutas e façam alterações visando à melhoria do programa baseado em evidências. 


\section{Conclusão}

O programa de controle ao tabagismo em Tubarão (SC) demonstrou uma efetividade similar a outros estudos.

A maioria dos usuários que participaram do programa foram mulheres de meia idade, entretanto, os usuários do sexo masculino obtiveram melhores resultados. Além disso, a dependência de nicotina e o uso de bupropiona não foram fatores determinantes no desfecho.

Foi observado também que as três primeiras semanas foram o período com melhores resultados na iniciativa dos usuários cessarem o tabagismo, e, ainda, aqueles que frequentaram mais que quatro encontros apresentaram melhores resultados sustentados ao final do período de tratamento.

\section{Referências}

1. World Health Organization - WHO. Global status report on noncommunicable diseases 2010: Description of the global burden of NCDs, their risk factors and determinants. Geneva: World Health Organization; 2011.

2. Silva LC, Araújo AJ, Queiroz AM, Sales MD, Castellano MV; Comissão de Tabagismo da SBPT. Smoking control: challenges and achievements. J Bras Pneumol. 2016;42(4):290-8. DOI: http://dx.doi.org/10.1590/s1806-37562016000000145

3. Oberg M, Jaakkola MS, Woodward A, Peruga A, Prüss-Ustün A. Worldwide burden of disease from exposure to second-hand smoke: a retrospective analysis of data from 192 countries. Lancet. 2011;377(9760):139-46. DOI: http://dx.doi.org/10.1016/S0140-6736(10)61388-8

4. Lightwood JM, Glantz SA. Declines in acute myocardial infarction after smoke-free laws and individual risk attributable to secondhand smoke. Circulation. 2009;120(14):1373-9. DOI: http://dx.doi.org/10.1161/CIRCULATIONAHA.109.870691

5. Shafey O, Ericksen M, Mackay J, Ross H. The Tobacco Atlas, 3rd ed. Atlanta: Bookhouse Group/American Cancer Society; 2009.

6. Brasil. Ministério da Saúde. Instituto Nacional de Câncer José Alencar Gomes da Silva (INCA). Convenção-Quadro para o Controle do Tabaco: Secretaria Executiva da Comissão Nacional para Implementação da Convenção-Quadro para o Controle do Tabaco. Rio de Janeiro: INCA; 2012.

7. Brasil. Ministério da Saúde. Instituto Nacional de Câncer José Alencar Gomes da Silva (INCA). Fundação Oswaldo Cruz (Fiocruz). O controle do tabaco no Brasil: uma trajetória. Rio de Janeiro: INCA; 2012.

8. Brasil. Ministério da Saúde. Tabagismo. Tratamento do tabagismo no SUS: informações aos gestores. Brasília: Ministério da Saúde; 2014.

9. Brasil. Ministério da Saúde. Instituto Nacional de Câncer José Alencar Gomes da Silva (INCA). Coordenação de Prevenção e Vigilância (CONPREV). Abordagem e Tratamento do Fumante: Consenso 2001. Rio de Janeiro: INCA; 2001.

10. Mesquita AA. Avaliação de um programa de tratamento do tabagismo. Rev Bras Ter Comport Cogn. 2013;15(2):35-44.

11. Azevedo RCS, Higa CMH, Assumpção ISMA, Frazatto CRG, Fernandes RF, Goulart W, et al. Grupo terapêutico para tabagistas: resultados após seguimento de dois anos. Rev Assoc Méd Bras. 2009;55(5):593-6. DOI: http://dx.doi.org/10.1590/S0104-42302009000500025

12. Meier DAP, Vannuchi MTO, Secco IAO. Abandono do tratamento do tabagismo em programa de município do norte do Paraná. Rev Esp Saúde. 2011;13(1):35-44.

13. Rodrigues NC, Neves RD, Mendes Dde S, Mendes CP, Martins IH, Reis IN, et al. Profile of Brazilian smokers in the National Program for Tobacco Control. Rev Bras Psiquiatr. 2015;37(2):150-4. DOI: http://dx.doi.org/10.1590/1516-4446-2014-1611

14. Peña P, Zagolin M, Acuña M, Navarrete S, Bustamante P, Canals A. Factors associated with success of a smoking cessation program. Rev Med Chil. 2016;144(8):965-71.

15. Mavrot C, Stucki I, Sager F, Etter JF. Efficacy of an Internet-based, individually tailored smoking cessation program: A randomizedcontrolled trial. J Telemed Telecare. 2017;23(5):521-8. DOI: http://dx.doi.org/10.1177/1357633X16655476 
16. Jesus MCP, Silva MH, Cordeiro SM, Kortchmar E, Zampier VSB, Merighi MAB. Understanding unsuccessful attempts to quit smoking: a social phenomenology approach. Rev Esc Enferm USP. 2016;50(1):71-8. DOI: http://dx.doi.org/10.1590/S0080-623420160000100010

17. Eckerdt NS, Corradi-Webster CM. Sentidos sobre o hábito de fumar para mulheres participantes de grupo de tabagistas. Rev Latino Am Enferm. 2010;18(no.spe):641-7. DOI: http://dx.doi.org/10.1590/S0104-11692010000700022

18. Figueiró LR, Barros HM, Ferigolo M, Dantas DC. Assessment of factors related to smokers' adherence to a short-term support group for smoking cessation: a longitudinal study in a developing country. Trends Psychiatry Psychother. 2017;39(1):19-28. DOI: http://dx.doi. org/10.1590/2237-6089-2016-0041

19. Curry SJ, Mermelstein RJ, Sporer AK. Sustainability of Community-Based Youth Smoking Cessation Programs: Results From a 3-Year Follow-Up. Health Promot Pract. 2016;17(6):845-52. DOI: http://dx.doi.org/10.1177/1524839916657326

20. França SAS, Neves ALF, Souza TAS, Martins MCN, Carneiro SR, Sarges ESNF, et al. Fatores associados à cessação do tabagismo. Rev Saúde Pública. 2015;49:10.

a Universidade do Sul de Santa Catarina (UNISUL). Tubarão, SC, Brasil. kelserkock@yahoo.com.br (Autor corrrespondente); matheuswalerio@gmail.com

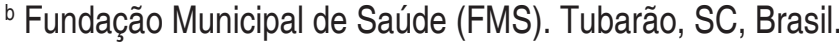
calouzinhac@ hotmail.com; solangecastelini@ hotmail.com 